\title{
High Prudent diet factor score predicts lower relapse hazard in early multiple sclerosis
}

Steve Simpson-Yap ${ }^{1,2}$, Wendy H. Oddy ${ }^{1}$, Bruce Taylor ${ }^{1}$, Robyn M Lucas ${ }^{3,4}$, Lucinda J Black $^{5}$, Anne-Louise Ponsonby ${ }^{3,6,7}$, Leigh Blizzard ${ }^{1}$, Ausimmune/AusLong Investigators Group $^{8}$, Ingrid van der $\mathrm{Mei}^{1}$

Institutions

1 Menzies Institute for Medical Research, University of Tasmania, Australia;

2 Neuroepidemiology Unit, Melbourne School of Population and Global Health, The University of Melbourne, Australia;

3 National Centre for Epidemiology and Population Health, Research School of Population Health, Australian National University, Australia;

4 Centre for Ophthalmology and Visual Science, University of Western Australia, Australia 5 School of Public Health, Curtin University, Australia;

6 Murdoch Children's Research Institute, The University of Melbourne, Australia;

7 Florey Department of Neuroscience and Mental Health, The University of Melbourne, Australia;

8 Full list of co-investigators' affiliations are listed in Acknowledgements.

Corresponding author

A/Professor Ingrid van der Mei

Menzies Institute for Medical Research, University of Tasmania

Private bag 23

Hobart, Tasmania

Australia 7000

ingrid.vandermei@utas.edu.au

Keywords: dietary patterns, multiple sclerosis, factor analysis, MS conversion, relapse

Abstract: 200/200

Body: 2953/3000

Refs: $32 / 35$

Tables: 4

Figures: 1

Supp Tables: 6 


\section{Abstract}

Background: Dietary patterns and their association with subsequent clinical course have not been well-studied in early MS.

Objectives: To describe dietary patterns in people in five years following first clinical demyelination, and assess associations with MS conversion and relapse.

Methods: Baseline food frequency questionnaire dietary intake (entry to the Ausimmune Study) and 5-year follow-up; iterated principal factor analysis applied. MS conversion and relapse risks assessed by Cox proportional hazards models, adjusted for age, sex, study site, education, BMI, smoking, and omega-3 supplement use.

Results: In cases with a first clinical diagnosis of CNS demyelination, we identified three major dietary patterns, 'Prudent, 'High-Vegetable', and 'Mixed', explaining 43\%, 37\% and $24 \%$ of diet variance in dietary intake. Fruits, vegetables, fish, wholegrains and nuts loaded highly on the Prudent pattern, starchy vegetables and legumes on the High-Vegetable pattern, and meats and alcohol on the Mixed pattern. Diet factor scores were not associated with MS conversion risk. Those with baseline Prudent scores above the median had significantly lower relapse risk (adjusted hazard ratio $=0.54,95 \%$ CI $0.37-0.81$ ) with some evidence of a plateau effect.

Conclusions: Prudent diet factor score above the median prospectively associated with lower relapse risk in five years following first clinical demyelinating event. 


\section{Abbreviations}

BMI Body Mass Index

CNS Central Nervous System

CIS Clinically Isolated Syndrome

FCD First Clinical Diagnosis of CNS demyelination

FFQ Food Frequency Questionnaire

HR Hazard ratio

MS Multiple sclerosis 


\section{Introduction}

While there has long been interest in the impacts of specific diets in multiple sclerosis $(\mathrm{MS})^{1-}$ ${ }^{3}$, there have been few well-conducted studies with prospective collection of diet and disease progression measures. Due to the complexity of the human diet, and of its measurement, the study of diet is challenging. Traditional approaches to analyses of diet and MS in observational studies have focused on specific foods and nutrients ${ }^{4-6}$. However, dietary pattern analysis takes account of the whole diet and the effect of food combinations, to explore associations between certain eating styles and determinants of disease progression in people with $\mathrm{MS}^{7}$. While prior studies have explored a relationship with dietary pattern and MS risk ${ }^{8}$, associations with disease progression have not been reported.

A better understanding of the determinants of diet factor scores could provide information for clinical management in this area. In addition, demonstrating prospective associations between patterns and clinical outcomes could guide dietary recommendations for people with MS and indicate points for intervention. The present study builds upon the work conducted in the Ausimmune Study ${ }^{8-11}$, which indicated a beneficial impact of healthy dietary intake in reducing the risk of MS onset, and to ascertain whether this effect is evident in relation to the disease course over five years of follow-up. The objectives of this study were to use a prospective cohort study to: 1) determine diet factor scores soon after first diagnosis of central nervous system (CNS) demyelination (baseline) using factor analysis; 2) investigate predictors of diet factor scores and change thereof between baseline and 5-year follow-up using diet data from baseline and 5-year reviews; and 3) test associations between diet factor scores and hazards of MS conversion and relapse. 


\section{Methods}

The Ausimmune Study was a population-based multicentre case-control study (2003-2006) conducted in four regions of eastern Australia: Brisbane, Newcastle and surrounds, Geelong and the Western Districts of Victoria, and Tasmania ${ }^{12}$. The cohort comprised individuals presenting with a first clinical diagnosis of CNS demyelination including 1: clinically isolated syndrome (CIS); 2: first clinical diagnosis of CNS demyelination, but, on specific questioning, had a previous undiagnosed demyelinating event; 3: initial diagnosis of progressive-onset MS. Since 2009, the Ausimmune Longitudinal (AusLong) Study has investigated risk factors for early MS progression in cases from the Ausimmune Study ${ }^{13}$, with annual telephone data collection and face-to-face interviews at $2 / 3$ years (retention $=94.3 \%$ ), and 5 years (retention $=84.6 \%)$.

Ethics committees of all participating centres approved both studies; all participants signed written informed consent.

\section{Food Frequency Questionnaire (FFQ)}

The self-administered Cancer Council Victoria Dietary Questionnaire for Epidemiological Studies version 2 assessed typical dietary intake for 101 foods and beverages over the previous year ${ }^{14}$. Nutrient intakes were estimated using Australian food composition data ${ }^{15}$.

\section{Clinical outcome measures}

First, analyses of diet factor scores were evaluated among all persons completing FFQs (at baseline with a first clinical diagnosis of CNS demyelination), to maximise generalisability to people with MS and to allow the application of diet factor scores to other outcomes in future studies. Then, the association between diet factor scores with MS conversion and relapse 
included only CIS cases. This CIS only group was used to minimise any possible diseaserelated influences on both choice of diet and the outcomes under study.

Within-study and historical clinical history were derived from questionnaire and neurological notes. MS conversion was defined as the occurrence of a second clinical demyelinating episode (96.6\%), or MRI evidence of a new lesion $(3.4 \%)^{16}$. Relapses were defined according to the 2001 McDonald Criteria $^{17}$; only diagnosed relapses were included in the analysis. All diagnosed relapses, including the first post-CIS relapse defining MS conversion and all subsequent events up to 5-year review, were included in relapse analyses.

\section{Other covariates}

Data were obtained from participants at face-to-face interviews as described previously ${ }^{18}$, including demographic characteristics, height and weight used to calculate BMI ${ }^{19}$, medication/supplement use (yes/no for vitamin D containing supplements, omega-3/6 containing supplements, use of immunomodulatory medications), smoking (never/past/current), education, employment, and other characteristics. Physical activity was assessed using a modified version of the International Physical Activity Questionnaire ${ }^{20}$.

\section{Statistical methods}

\section{Factor analysis}

FFQ items were grouped into 34 groups devised a priori (Supplemental Table 1$)^{8,21}$. We excluded participants with implausible energy intakes of $<3000$ or $>20,000$ kilojoules/day $(\mathrm{n}=15)$, as per prior research ${ }^{21,22}$. Of note, application of a more stringent energy intake at bounds of the $25^{\text {th }}$ percentile minus the interquartile range and the $75^{\text {th }}$ percentile plus the interquartile range as described previously ${ }^{23}$ produced similar factors and factor loadings 
(data not shown). Food intake data were adjusted for energy using the residual method ${ }^{24}$, whereby food groups were adjusted for energy intake and the residuals from this model defining the food groups used in the factor analyses.

Iterated principal-factor analysis with oblique rotation ${ }^{7}$ was applied to the baseline food intake data to determine dietary factor scores, and these were used as predictors of MS conversion and relapse. In addition, iterated principal-factor analysis with oblique rotation was applied using dietary intake data from baseline and 5-year follow-up, estimating factor scores at each timepoint from a single model. Factor scores from this combined analysis were used to evaluate change in factor scores between the baseline and 5-year follow-up. The Bartlett test of sphericity was significant for both models $(\mathrm{p}<0.001)$, supporting sampling adequacy. Food groups having a factor loading $\geq|0.20|$ were considered important contributors to each pattern. Individual participant diet factor scores at baseline and 5-year follow-up were predicted using the Bartlett method ${ }^{25}$.

\section{Statistical analysis}

Relationships of nutrient content with diet factor scores were assessed using univariable linear regression. Characteristics of baseline dietary pattern scores were investigated using linear regression, adjusted for age, sex, and study site. Predictors of changes in diet factor scores were investigated using linear regression, adjusting for age, sex, study site, and baseline dietary factor score. Further adjusted models included education, baseline BMI, baseline current smoking, and baseline use of omega-3/6-containing supplements. In addition, to allow comparability with the CIS-only clinical outcome analyses, characteristics of baseline diet factor scores and change thereof were evaluated constrained to CIS-only (Supplementary Tables 4 \& 6). 
Predictors of time to MS conversion and of relapse were evaluated by Cox proportionalhazards regression, relapse models with shared frailties, estimating hazard ratios (HR) and adjusted hazard ratios (aHR). Diet factor scores were evaluated first as continuous terms, and then to examine potential threshold/plateau effects, diet factor scores were categorised into quartiles. MS conversion and relapse models were adjusted for age, sex, and study site, and further adjusted for baseline BMI, baseline smoking, and baseline omega-3/6 supplement use. Further adjustment for baseline physical activity and immunomodulatory medication use had no material impact (data not shown). Time-at-risk for MS conversion and relapse began from the referral episode date. All covariates satisfied the proportional hazards assumption except study site for relapse, so models were estimated including products terms of study site and time.

For all analyses, p-values for tests for trend for ordinal polytomous variables were calculated by replacing the dummy dichotomous variables of the polytomous variable with a single variable, thus estimating the significance of the difference from 1 of a linear trendline across the levels of the polytomous variable.

Statistical analyses were conducted in Stata/SE 15.1 (StataCorp LP, College Station, USA).

\section{Results}

Baseline and 5-year FFQs were completed by $96 \%$ of participants with a first clinical diagnosis of CNS demyelination (268/279, and 227/236, respectively; Table 1) and 209 participants completed FFQs at both timepoints. Over half of the participants were overweight/obese and most had low physical activity. Baseline characteristics were generally similar for those with and without FFQ data. However, current smokers were significantly less likely to have completed an FFQ at both timepoints. Amongst eligible participants with a CIS only, 159/170 completed the baseline FFQ and 137/145 the 5-year follow-up FFQ; 128 
CIS participants completed FFQs at both baseline and follow-up. Associations with covariates at baseline and predictors of change in score from baseline to 5-year follow-up for CIS were similar to those for the full participant group (with a first clinical diagnosis of CNS demyelination, Supplemental Tables 4 \& 6).

\section{Characteristics of dietary patterns}

Three factors were identified ("Prudent", "High-Vegetable", and "Mixed") using the baseline-only FFQ, which explained $43 \%, 37 \%$, and $24 \%$ of the post-rotation variances (Table 2). Foods loading positively on the Prudent diet factor score included fresh fruit, nonfried fish, nuts, and leafy vegetables. Foods loading positively on the High-Vegetable diet factor scores included yellow/red and cruciferous vegetables, legumes, potatoes, and sauce. Foods loading positively on the Mixed diet factor score included poultry, red and processed meat, and alcohol. Similar characteristics were seen at baseline for the combined (baseline and 5-year follow-up) factor analysis used for analyses of change in diet factor scores (Supplemental Table 2).

The nutritional components of the diet substantiated the characterisations of the diet patterns, such that healthy nutrients were positively associated with Prudent and High-Vegetable and unhealthy with Mixed diet factor scores (Supplemental Table 3). The Prudent diet factor score was positively associated with omega-3, magnesium, potassium, iron and vitamins $\mathrm{C}$ and $\mathrm{E}$, but negatively with total and saturated fats. High-Vegetable diet factor score was positively associated with magnesium, potassium, and vitamins $\mathrm{C}$ and $\mathrm{E}$, but negatively with cholesterol. Mixed diet factor score was positively associated with protein, cholesterol, and zinc, but negatively with carbohydrates, sugars, and vitamin E. 


\section{Covariates associated with baseline diet factor scores}

As in Table 3, baseline Prudent diet factor score was significantly and independently higher among participants in the oldest age quartile, among the more physically active, those taking omega-3/6 supplements, and those who had completed university, but lower among overweight participants. Current smokers and those engaged in physical labour had lower Prudent diet factor scores, but these associations attenuated and became non-significant on adjustment. High-Vegetable diet factor score was significantly lower among current smokers and among those who had completed TAFE/trade school. Mixed diet factor score was significantly higher among ex and current smokers, and among those who had completed university but was lower among the non-employed. No diet factor scores were significantly and independently associated with age, sex, vitamin D supplement use, MS onset type, or immunomodulatory medication use. Results were not materially different on restricting to CIS cases (Supplemental Table 4).

\section{Change in diet factor scores (baseline to 5-year follow-up) and characteristics thereof}

There was little change in diet factor scores during follow-up, average Prudent diet factor score increasing only 0.02 and High-Vegetable 0.06, while Mixed decreased by 0.04 (Supplemental Table 5). Those who were obese at baseline review showed a significantly greater increase in Prudent diet factor score. However, no other characteristics were significantly and robustly associated with subsequent change in diet factor scores. Results were not materially different on restricting to CIS cases (Supplemental Table 6).

\section{Diet and clinical outcomes}

MS conversion - No baseline diet factor scores were associated with MS conversion hazard, nor was there any consistent dose-dependency suggestive of trend (Table 4). 
Relapse hazard - As a continuous term, a one-unit higher Prudent score showed an inverse trend, with a $13 \%$ lower hazard of relapse $(\mathrm{p}=0.11)$, persisting in magnitude on further adjustment $(\mathrm{aHR}=0.85, \mathrm{p}=0.09)$. As a quartile categorical term this reached significance ( $\left.\mathrm{p}_{\text {trend }}=0.034\right)$, albeit of mixed dose-dependency, and consolidating the lower two levels showed evidence of a plateau effect, such that those in the top two levels had $52 \%$ and $40 \%$ lower relapse risk $\left(\mathrm{p}_{\text {trend }}=0.017\right)$ and those above the median score had $46 \%$ lower relapse risk relative to the bottom $50 \%(\mathrm{p}=0.002$, Figure 1). Neither High-Vegetable nor Mixed diet factor scores showed any associations with relapse risk.

\section{Discussion}

Diet is a modifiable lifestyle factor which people with MS may alter with the aim of improving their prognosis. In this prospective study of people with early MS, we provide evidence of three dietary patterns that we labelled, "Prudent, "High-Vegetable", and "Mixed". Foods loading strongly on each factor, their nutritional characteristics, and baseline characteristics of participants were generally in line with the labels ascribed to each pattern. Over the 5-year follow-up, there was little change in diet factor scores. In participants with a CIS at baseline, no diet factor scores were significantly associated with subsequent risk of MS conversion. However, higher Prudent diet factor score was associated with reduced relapse risk, such that those above the median had a $46 \%$ lower relapse risk.

Others have examined the association of dietary intake with MS and clinical progression ${ }^{4-6,26 \text {, }}$ 27 , including using factor analysis or related methods ${ }^{8,28}$. The AusLong cohort has previously examined a range of factors for their associations with clinical progression in early MS, ranging from sun and vitamin D metabolite $\operatorname{levels}^{29}$, BMI and dyslipidaemia ${ }^{8,28}$, tobacco and marijuana smoking ${ }^{8}$, and genetic risk factors ${ }^{2,30}$. Also, the Ausimmune case-control study ${ }^{13}$ from which the AusLong Study derives, has explored a range of lifestyle factors, including $\operatorname{diet}^{8-11,26}$. Our results are in keeping with the previous Ausimmune findings that a healthy 
$\operatorname{diet}^{8}$, particularly one rich in fish ${ }^{11}$, was inversely associated with case status. That our present study did not show a significant association between Prudent diet factor score and MS conversion risk contrasts with these results, although the focus of that analysis was a first clinical diagnosis of CNS demyelination, rather than MS conversion. Also, here these analyses were restricted to CIS cases. Moreover, whereas the Ausimmune analyses based their factor analysis on the healthy control population, leading to the estimation of two factors, one Healthy and one Western, our factor analysis used dietary intake from the cases and led to three generally healthy diets. Thus, while the healthiest diet pattern in this sample, which we labelled Prudent, was inversely associated with relapse risk, neither of the other two patterns was distinctly deleterious.

The results seen for Prudent diet factor score and relapse are promising as they suggest a potential point of intervention whereby a better quality of diet may be beneficial in reducing clinical progression. Although we did not see a significant association of Prudent diet factor score with MS conversion risk, there was an inverse trend, albeit one of inconsistent dosedependency. The two outcomes in this sample are related - the MS conversion event is the first post-CIS relapse for the majority of the sample (where the diagnosis was based on a second clinical episode). Thus, we would hope for internal consistency that a true association would show a signal for both MS conversion and relapse, despite the lower event numbers for MS conversion which limits statistical power compared to relapse. However, as noted, the dietary behaviour of the whole cohort was generally healthy in nature, the worst being "Mixed" in character. It may be that the healthiest of these behaviours might have a longterm accrued benefit against the inflammatory processes underlying both MS conversion and relapse but not one which as strongly protects against the initial diagnostic event. 
Participants largely did not change their diet factor scores during follow-up diet factor score. Although those of obese BMI at baseline were significantly more likely to increase their Prudent diet factor score compared to normal BMI participants, no other characteristics were significantly associated with changes in diet factor scores. Using the case-control Ausimmune Study, Russell and colleagues examined determinants of change in dietary intake following a first clinical diagnosis of CNS demyelination $(\mathrm{FCD})^{31}$, finding no significant demographic or behavioural determinants, which is in line with our results.

Several advantages exist for analysing dietary intake as diet factor score, including taking account of whole diet, overcoming collinearity between nutrients, and decreasing the number of statistical tests needed to model disease hazard ${ }^{7}$. We acknowledge the limitation of FFQs in that some respondents underestimate intake. They are also subject to some recall error in contrast to more proximal 24-hour recall instruments. Nonetheless, FFQs remain one of the most practical dietary methods for epidemiological studies. While our sample size is somewhat small, this is balanced by a robust, prospective longitudinal study design, including the measurement of dietary intake in a consistent and systematic fashion at baseline and follow-up, and the thorough assessment of relapse and clinical activity, both by questionnaire, study neurological review, and review of neurological notes. A further limitation is that we assessed diet only at baseline and 5-year timepoints. It is possible that dietary changes through years 1-4 may have altered the risk of MS conversion or relapse, although our analyses show little change in diet from baseline to 5 years. Finally, those with more active disease may have had more motivation to change their diet in some fashion, and some medications like Tecfidera can impact on appetite, potentially leading to changes in diet. 
The Ausimmune Study aimed to recruit all eligible cases within the study regions ${ }^{13}$, and there was high participation amongst eligible $\operatorname{cases}^{32}$. The AusLong Study had excellent retention at 5 years. Thus, the cohort is representative of the cases who had a first clinical diagnosis of CNS demyelination between 2003 and 2006 from the study regions, supporting the generalisability of these findings. Importantly, in recruiting a sample soon after referral for a clinically isolated syndrome potential behaviour change secondary to disease is unlikely.

In conclusion, we have reproducibly shown three dietary patterns, a healthy Prudent, and two other patterns, one High-Vegetable and the other Mixed, which characterised the dietary intake of the cohort over five years of follow-up. These patterns are substantiated by their food group components, their agreement with nutritional and behavioural characteristics, and are comparable to those recently described using the baseline portion of this cohort ${ }^{8}$. We found that high baseline Prudent diet factor score was associated with a subsequent lower relapse risk, but not associated with MS conversion risk. Given the great public interest in diet and its potential role in MS onset and progression, its modifiable nature, as well as biological plausibility for the role of diet in MS, further investigation of the impact of diet on MS clinical course is warranted. 


\section{Ethics}

This study was conducted according to the guidelines laid down in the Declaration of Helsinki and all procedures involving human subjects were approved by nine regional human research ethics committees. Written informed consent was obtained from all subjects.

\section{Transparency declaration}

The lead author affirms that this manuscript is an honest, accurate, and transparent account of the study being reported. The reporting of this work is compliant with STROBE guidelines. The lead author affirms that no important aspects of the study have been omitted and that any discrepancies from the study as planned have been explained.

\section{Acknowledgements}

We express our heartfelt thanks to the participants in the Ausimmune and AusLong studies for their time and energy, without which we could not have realized this work.

We sincerely acknowledge the outstanding input of research personnel and research officers working on the studies.

The members of the Ausimmune/AusLong Investigators Group are as follows: Robyn M Lucas (National Centre for Epidemiology and Population Health, Canberra), Keith Dear (University of Adelaide, Australia), Anne-Louise Ponsonby and Terry Dwyer (Murdoch Childrens Research Institute, Melbourne, Australia), Ingrid van der Mei, Leigh Blizzard, Steve Simpson-Yap and Bruce V Taylor (Menzies Institute for Medical Research, University of Tasmania, Hobart, Australia), Simon Broadley (School of Medicine, Griffith University, Gold Coast Campus, Australia), Trevor Kilpatrick (Centre for Neurosciences, Department of Anatomy and Neuroscience, University of Melbourne, Melbourne, Australia). David 
Williams and Jeanette Lechner-Scott (University of Newcastle, Newcastle, Australia),

Cameron Shaw and Caron Chapman (Barwon Health, Geelong, Australia), Alan Coulthard

(University of Queensland, Brisbane, Australia), Michael P Pender (The University of

Queensland, Brisbane, Australia) and Patricia Valery (QIMR Berghofer Medical Research

Institute, Brisbane, Australia).

\section{Funding}

The Ausimmune and AusLong Studies were funded by the National Multiple Sclerosis

Society of the United States of America (award RG3364A1/2) and the National Health and

Medical Research Council of Australia (APP316901 and 224215). LJB is supported by an

MS Research Australia Postdoctoral Fellowship and a Curtin University Research

Fellowship.

\section{Data access}

Persons desiring access to the data underlying this report may contact the head of the MS

Research Group at the Menzies Institute for Medical Research, University of Tasmania,

Professor Bruce Taylor: bruce.taylor@utas.edu.au.

\section{References}

1. Swank RL and Dugan BB. Effect of low saturated fat diet in early and late cases of multiple sclerosis. Lancet (London, England). 1990; 336: 37-9.

2. Yadav V, Marracci G, Kim E, et al. Low-fat, plant-based diet in multiple sclerosis: A randomized controlled trial. Multiple sclerosis and related disorders. 2016; 9: 80-90.

3. Wahls T, Scott MO, Alshare Z, et al. Dietary approaches to treat MS-related fatigue: comparing the modified Paleolithic (Wahls Elimination) and low saturated fat (Swank) diets on perceived fatigue in persons with relapsing-remitting multiple sclerosis: study protocol for a randomized controlled trial. Trials. 2018; 19: 309.

4. Schwarz S and Leweling H. Multiple sclerosis and nutrition. Multiple sclerosis. 2005; 11: 2432. 
5. Jelinek GA, Hadgkiss EJ, Weiland TJ, Pereira NG, Marck CH and van der Meer DM. Association of fish consumption and Omega 3 supplementation with quality of life, disability and disease activity in an international cohort of people with multiple sclerosis. The International journal of neuroscience. 2013; 123: 792-800.

6. Fitzgerald KC, Tyry T, Salter A, et al. A survey of dietary characteristics in a large population of people with multiple sclerosis. Multiple sclerosis and related disorders. 2018; 22: 12-8.

7. Bartholomew DJ, Knott M and Moustaki I. Latent Variable Models and Factor Analysis: A unified approach. 3rd edition. 3rd ed. London: John Wiley \& Sons, Ltd., 2011.

8. Black LJ, Rowley C, Sherriff J, Pereira G, Ponsonby AL and Lucas RM. A healthy dietary pattern associates with a lower risk of a first clinical diagnosis of central nervous system demyelination. Multiple sclerosis (Houndmills, Basingstoke, England). 2018; 25: 1514-25.

9. Black LJ, Baker K, Ponsonby AL, van der Mei I, Lucas RM and Pereira G. A Higher Mediterranean Diet Score, Including Unprocessed Red Meat, Is Associated with Reduced Risk of Central Nervous System Demyelination in a Case-Control Study of Australian Adults. The Journal of nutrition. 2019.

10. Black LJ, Bowe GS, Pereira G, et al. Higher Non-processed Red Meat Consumption Is Associated With a Reduced Risk of Central Nervous System Demyelination. Frontiers in neurology. 2019; 10: 125.

11. Black LJ, Zhao Y, Peng YC, et al. Higher fish consumption and lower risk of central nervous system demyelination. European journal of clinical nutrition. 2019: doi: 10.1038/s41430-019-0476-z. 12. Lucas R, Ponsonby AL, Dear K, et al. Associations between silicone skin cast score, cumulative sun exposure and other factors in the Ausimmune Study: a multicentre Australian study. Cancer Biomarker Prevention 2009; 18: 2887-94.

13. Lucas R, Ponsonby AL, McMichael A, et al. Observational analytic studies in multiple sclerosis: controlling bias through study design and conduct. The Australian Multicentre Study of Environment and Immune Function. Multiple sclerosis (Houndmills, Basingstoke, England). 2007; 13: 827-39.

14. Giles G and Ireland P. Dietary Questionnaire for Epidemiological Studies (Version 2). Melbourne: Cancer Council Victoria, 1996.

15. Food Standards Australia New Zealand. NUTTAB 2010:

http://www.foodstandards.gov.au/science/monitoringnutrients/nutrientables/Pages/default.aspx. Food Standards Australia New Zealand, 2018.

16. Polman CH, Reingold SC, Banwell B, et al. Diagnostic criteria for multiple sclerosis: 2010 revisions to the McDonald criteria. Annals of neurology. 2011; 69: 292-302.

17. McDonald WI, Compston A, Edan G, et al. Recommended diagnostic criteria for multiple sclerosis: guidelines from the International Panel on the diagnosis of multiple sclerosis. Annals of neurology. 2001; 50: 121-7.

18. Ponsonby AL, Lucas RM, Dear K, et al. The physical anthropometry, lifestyle habits and blood pressure of people presenting with a first clinical demyelinating event compared to controls: The Ausimmune study. Multiple sclerosis (Houndmills, Basingstoke, England). 2013; 19: 1717-25. 19. Tettey P, Simpson S, Taylor B, et al. An adverse lipid profile and increased levels of adiposity significantly predict clinical course after a first demyelinating event. Journal of neurology, neurosurgery, and psychiatry. 2017; 88: 395-401.

20. Craig CL, Marshall AL, Sjostrom M, et al. International physical activity questionnaire: 12country reliability and validity. Medicine and science in sports and exercise. 2003; 35: 1381-95.

21. Ambrosini GL, Fritschi L, de Klerk NH, Mackerras D and Leavy J. Dietary patterns identified using factor analysis and prostate cancer risk: a case control study in Western Australia. Annals of epidemiology. 2008; 18: 364-70.

22. Rockett HR, Breitenbach M, Frazier AL, et al. Validation of a youth/adolescent food frequency questionnaire. Preventive medicine. 1997; 26: 808-16.

23. Flood A, Rastogi T, Wirfalt E, et al. Dietary patterns as identified by factor analysis and colorectal cancer among middle-aged Americans. The American journal of clinical nutrition. 2008; 88: $176-84$.

24. Willett WC, Howe GR and Kushi LH. Adjustment for total energy intake in epidemiologic studies. The American journal of clinical nutrition. 1997; 65: 1220S-8S; discussion 9S-31S. 
25. Bartlett MS. The statistical conception of mental factors. British Journal of Psychology. 1937;

28.

26. Hoare S, Lithander F, van der Mei I, Ponsonby AL and Lucas R. Higher intake of omega-3 polyunsaturated fatty acids is associated with a decreased risk of a first clinical diagnosis of central nervous system demyelination: Results from the Ausimmune Study. Multiple sclerosis (Houndmills, Basingstoke, England). 2016; 22: 884-92.

27. Sedaghat F, Jessri M, Behrooz M, Mirghotbi M and Rashidkhani B. Mediterranean diet adherence and risk of multiple sclerosis: a case-control study. Asia Pacific journal of clinical nutrition. 2016; 25: 377-84.

28. Jahromi SR, Toghae M, Jahromi MJ and Aloosh M. Dietary pattern and risk of multiple sclerosis. Iranian journal of neurology. 2012; 11: 47-53.

29. Simpson Jr. SL, van der Mei I, Lucas R, et al. Sun exposure across the life course significantly modulates early multiple sclerosis clinical course. Frontiers in neurology. 2018; 9: doi: 10.3389/fneur.2018.00016.

30. Bisht B, Darling WG, Grossmann RE, et al. A multimodal intervention for patients with secondary progressive multiple sclerosis: feasibility and effect on fatigue. Journal of alternative and complementary medicine (New York, NY). 2014; 20: 347-55.

31. Russell RD, Lucas RM, Brennan V, Sherriff JL, Begley A and Black LJ. Reported Changes in Dietary Behavior Following a First Clinical Diagnosis of Central Nervous System Demyelination. Frontiers in neurology. 2018; 9: 161.

32. Lucas RM, Ponsonby AL, Dear K, et al. Sun exposure and vitamin D are independent risk factors for CNS demyelination. Neurology. 2011; 76: 540-8. 
Figure 1. Kaplan-Meier plot of baseline Prudent diet score (above vs below median) vs subsequent hazard of relapse. Note: $100 \%$ survival indicates the whole cohort being relapse-free, which only occurs at baseline. 